\title{
Balanced versus chloride-rich solutions for fluid resuscitation in brain-injured patients: a randomised double-blind pilot study
}

Antoine Roquilly ${ }^{1 \dagger}$, Olivier Loutrel ${ }^{1 \dagger}$, Raphael Cinotti ${ }^{2}$, Elise Rosenczweig ${ }^{3}$, Laurent Flet ${ }^{4}$, Pierre Joachim Mahe ${ }^{1}$, Romain Dumont ${ }^{1}$, Anne Marie Chupin ${ }^{1}$, Catherine Peneau', Corinne Lejus ${ }^{1}, Y_{\text {vonnick Blanloeil }}^{2}$, Christelle Volteau ${ }^{5}$ and Karim Asehnoune ${ }^{1 *}$

\begin{abstract}
Introduction: We sought to investigate whether the use of balanced solutions reduces the incidence of hyperchloraemic acidosis without increasing the risk for intracranial hypertension in patients with severe brain injury.

Methods: We conducted a single-centre, two-arm, randomised, double-blind, pilot controlled trial in Nantes, France. Patients with severe traumatic brain injury (Glasgow Coma Scale score $\leq 8$ ) or subarachnoid haemorrhage (World Federation of Neurosurgical Society grade III or higher) who were mechanically ventilated were randomised within the first 12 hours after brain injury to receive either isotonic balanced solutions (crystalloid and hydroxyethyl starch; balanced group) or isotonic sodium chloride solutions (crystalloid and hydroxyethyl starch; saline group) for 48 hours. The primary endpoint was the occurrence of hyperchloraemic metabolic acidosis within 48 hours.
\end{abstract}

Results: Forty-two patients were included, of whom one patient in each group was excluded (one consent withdrawn and one use of forbidden therapy). Nineteen patients (95\%) in the saline group and thirteen (65\%) in the balanced group presented with hyperchloraemic acidosis within the first 48 hours (hazard ratio $=0.28,95 \%$ confidence interval $[\mathrm{Cl}]=0.11$ to $0.70 ; P=0.006)$. In the saline group, $\mathrm{pH}(P=.004)$ and strong ion deficit $(P=0.047)$ were lower and chloraemia was higher $(P=0.002)$ than in the balanced group. Intracranial pressure was not different between the study groups (mean difference $4 \mathrm{mmHg}[-1 ; 8] ; P=0.088$ ). Seven patients $(35 \%)$ in the saline group and eight (40\%) in the balanced group developed intracranial hypertension $(P=0.744)$. Three patients (14\%) in the saline group and five (25\%) in the balanced group died $(P=0.387)$.

Conclusions: This study provides evidence that balanced solutions reduce the incidence of hyperchloraemic acidosis in brain-injured patients compared to saline solutions. Even if the study was not powered sufficiently for this endpoint, intracranial pressure did not appear different between groups.

Trial registration: EudraCT 2008-004153-15 and NCT00847977

The work in this trial was performed at Nantes University Hospital in Nantes, France.

\section{Introduction}

Brain injuries remain a major concern for public health services, particularly because of the high mortality rate and long-term disabilities that result [1]. In the early stages of caring for brain-injured patients, therapies are

\footnotetext{
* Correspondence: karim.asehnoune@chu-nantes.fr

+ Contributed equally

'Pôle Anesthésie-Réanimations, Service d'anesthésie réanimation Hôtel-Dieu, CHU Nantes, F-44000 Nantes, France

Full list of author information is available at the end of the article
}

focused on minimising secondary brain injuries that are centrally involved in determining outcomes [2]. Intracranial hypertension $(\mathrm{ICH})$ is the most frequent cause of death and secondary brain insults after brain injury [3]. The maintenance of adequate cerebral perfusion pressure (CPP), which is associated with control of intracranial pressure (ICP), is the cornerstone of treating the ion deficit associated with brain ischaemia in brain-injured patients. Infusion of hypo-osmotic solutions, which increases cerebral swelling, should be avoided after brain 
injury $[4,5]$. Current recommendations are to use isotonic solutions in patients with severe brain injury [6,7], with isotonic sodium chloride ( $0.9 \%$ saline solution) being the mainstay of therapy. Isotonic sodium chloride solutions induce hyperchloraemic metabolic acidosis and have side effects including haemostatic alterations, cognitive dysfunction and ileus [8].

Hyperchloraemia is relatively common in critically ill patients, and it is now commonly accepted that chloriderich fluids are the primary cause of hyperchloraemic acidosis in critically ill patients [9]. In a before-after study, a chloride-restrictive strategy was associated with a significant decrease in renal failure in critically patients and significantly affected electrolyte and acid-base status [10]. In a post hoc analysis of a retrospective study in TBI patients receiving isotonic sodium chloride solutions for basal infusion [11], 65\% of the patients experienced hyperchloraemia. Chloride channels regulate cell oedema [12], and it could be hypothesised that dyschloraemia contributes to brain swelling.

Isotonic balanced solutions are now available and include crystalloids as well as hydroxyethyl starch (HES) solutions. In these isotonic solutions, the use of malate and acetate allows the reduction of chloride concentration while ensuring isotonicity. Balanced solutions could thus reduce the incidence of hyperchloraemic metabolic acidosis. Balanced solutions decrease the rate of hyperchloraemic acidosis in healthy volunteers $[13,14]$ and during perioperative care compared with saline solutions [15-17]. To date, no data regarding isotonic balanced solutions for brain-injured patients have been published, and use of these solutions is therefore not recommended in this setting. The use of a balanced solution would appear to be attractive in brain-injured patients who are prone to ion homeostasis disruption, notably through hormonal dysfunction such as diabetes insipidus or cerebral salt-wasting syndrome or through alterations of chloride-dependent channels such as the NKCC1 transporter $[18,19]$. We postulated that infusion of isotonic balanced solutions instead of saline solutions would diminish the incidence of hyperchloraemic acidosis without increasing ICP in patients with severe brain injury hospitalised in the ICU.

\section{Materials and methods}

\section{Ethical approval and study design}

This randomised, double-blind, parallel, controlled study was approved by the Institutional Review Board of Tours, France (Région Centre, Ouest-1) (Trial registration: EudraCT 2008-004153-15 and NCT00847977). Patients were enrolled after their next-of-kin provided written informed consent. Retrospective consent, when available, was obtained from patients. Patients were enrolled from October 2008 to October 2010, when recruitment was completed in three ICUs of the Nantes University Hospital.

\section{Patient population}

Patients with severe traumatic brain injury (TBI) (Glasgow Coma Scale score $\leq 8$ ) on mechanical ventilation within the first 12 hours after brain injury were included. During recruitment, we refined the eligibility criteria by including patients with subarachnoid haemorrhage (SAH) at World Federation of Neurosurgical Societies (WFNS) grade III or worse (amendment of 26 July 2010). Exclusion criteria were multiple trauma, pregnancy, azotaemia above 200 $\mu \mathrm{mol} / \mathrm{L}$, kalemia less than $2.5 \mathrm{mmol} / \mathrm{L}$, calcaemia less than $1.8 \mathrm{mmol} / \mathrm{L}$, HES hypersensitivity, haemophilia or von Willebrand disease. Patients were also excluded when hypertonic saline solutions (HSSs) were used prior to inclusion or within the first 6 hours of the study start.

\section{Randomisation}

Patients were randomised in a 1:1 ratio to either the balanced group (allocated solutions, crystalloids: Isofundine/HES: Tetraspan; B Braun Medical, Melsungen, Germany) or the saline group (allocated solutions, crystalloids: $0.9 \%$ saline solution/HES: HEAfusine, B Braun Medical) (Table 1). Randomisation was performed in blocks of eight by a computerised number generator list provided by a statistician not involved in the determination of eligibility or in the assessment of outcomes. The study packs were sealed in identical sequentially numbered boxes containing the entire treatment for each patient. Each "Iso-TC treatment packet" contained Isofundine or $0.9 \%$ saline solution (sheath labelled "crystalloid"), Tetraspan or HEAfusine (sheath labelled "HES"), and a sheet was also provided for the administration schedule. Patients, investigators, members of the monitoring board and medical and nursing staff were unaware of the patients' treatment assignment.

\section{Conduct of the study}

Administration of the studied solutions began immediately after patient admission and lasted 48 hours. The attributed crystalloid was administered as a continuous intravenous infusion $(30 \mathrm{ml} / \mathrm{kg} /$ day $)$. The attending physician could administer optional boli $(20 \mathrm{ml} / \mathrm{kg}$ of the attributed crystalloid or $10 \mathrm{ml} / \mathrm{kg}$ of the attributed HES over 20 minutes). Apart from blood products, other intravenous fluids were not allowed during the first 48 hours. After the 48th hour, fluid infusions were not controlled.

\section{General care for brain-injured patients}

Brain-injured patients were mechanically ventilated and were sedated with fentanyl and midazolam (0.9\% saline solution as drug-carrier solution). Patients were kept in a semirecumbent position. Continuous enteral nutrition was initiated 24 hours after brain injury [20]. The rate of enteral nutrition (Fresubin; Fresenius-Kabi, France) was increased every 8 hours until it reached $83 \mathrm{ml} / \mathrm{h}$ 
Table 1 Electrolyte composition of studied fluids.

\begin{tabular}{|c|c|c|}
\hline & Saline group & $\begin{array}{l}\text { Balanced } \\
\text { group }\end{array}$ \\
\hline Crystalloid solutions & $\begin{array}{l}0.9 \% \text { saline } \\
\text { solution }\end{array}$ & Isofundine \\
\hline Sodium (mmol/L) & 153 & 140 \\
\hline Potassium (mmol/L) & 0 & 4.0 \\
\hline Calcium (mmol/L) & 0 & 2.5 \\
\hline Magnesium (mmol/L) & 0 & 1.0 \\
\hline Chloride mmol/L) & 153 & 127 \\
\hline Acetate $(\mathrm{mmol} / \mathrm{L})$ & 0 & 24 \\
\hline Malate (mmol/L) & 0 & 5.0 \\
\hline $\mathrm{pH}$ & 4 to 7 & 4.6 to 5.4 \\
\hline $\begin{array}{l}\text { Theoretical osmolarity } \\
\text { (mOsmol/L) }\end{array}$ & 306 & 304 \\
\hline Acid titre & $<2$ & $<2$ \\
\hline Hydroxyethyl starch solutions & HEAfusine & Tetraspan \\
\hline $\begin{array}{l}\text { Poly(O-2-hydroxyethyl) starch } \\
\text { (g/L) }\end{array}$ & 60 & 60 \\
\hline Molar substitution & 0.5 & 0.42 \\
\hline Average molecular weight (Da) & 200,000 & 130,000 \\
\hline Sodium (mmol/L) & 153 & 140 \\
\hline Potassium (mmol/L) & 0 & 4.0 \\
\hline Calcium (mmol/L) & 0 & 2.5 \\
\hline Magnesium (mmol/L) & 0 & 1.0 \\
\hline Chloride (mmol/L) & 153 & 118 \\
\hline Acetate $(\mathrm{mmol} / \mathrm{L})$ & 0 & 24 \\
\hline Malate (mmol/L) & 0 & 5.0 \\
\hline $\mathrm{pH}$ & 4 to 7 & 5.6 to 6.4 \\
\hline $\begin{array}{l}\text { Theoretical osmolarity } \\
\text { (mOsmol/L) }\end{array}$ & 310 & 296 \\
\hline Acid titre & $<2$ & $<2$ \\
\hline
\end{tabular}

(2,000 kcal/day) (see Additional file 1 for full description). Parenteral nutrition was started on day 7 in patients intolerant to gastric feeding. Secondary brain injuries were prevented by avoiding hypoxaemia and anaemia (haemoglobin $<10 \mathrm{~g} / \mathrm{dl}$ ), maintaining body temperature between $36.0^{\circ} \mathrm{C}$ and $37.0^{\circ} \mathrm{C}$, ensuring normoglycaemia and normocapnia (between 4.6 and $5.5 \mathrm{kPa}$ ). ICP was monitored with an intraparenchymal probe placed in the most affected side (Codman; Johnson \& Johnson, Raynham, MA, USA) in patients with severe brain injuries who had abnormal computed tomography (CT) scans and were considered at increased risk of ICH [21]. Extraventricular drainage was used in case of hydrocephalus detected on CT scans. Patients were monitored by invasive arterial pressure and mean arterial pressure (MAP) was measured up to the brain for the calculation of CPP. CPP was maintained above $60 \mathrm{mmHg}$ with boli of the attributed isotonic solutions (crystalloid or HES; see Table 1) and continuous infusion of norepinephrine (diluted in $0.9 \%$ saline solution). Mannitol (bolus of $0.5 \mathrm{~g} / \mathrm{kg}$ repeatable once in case of poor ICP control, ICP $>20 \mathrm{mmHg}$, after 30 minutes; maximum dose: $1 \mathrm{~g} / \mathrm{kg}$ ) was used to control episodes of $\mathrm{ICH}$. When control of $\mathrm{ICH}$ was poor, sodium thiopental was used with a loading dose ( 2 to $3 \mathrm{mg} / \mathrm{kg}$ ) followed by continuous administration ( 2 to $3 \mathrm{mg} / \mathrm{kg} / \mathrm{h}$ ) adapted to ICP evolution and to serum level monitoring (blood level of thiopental between 20 and $30 \mu \mathrm{g} / \mathrm{ml}$ ). A continuous infusion of HSS (20\% saline solution) was started in case of refractory ICH [11]. When control of ICH was poor, decompressive craniectomy or therapeutic hypothermia was discussed with the neurosurgical team. The evolution of brain injuries was assessed by $\mathrm{CT}$ within the first 72 hours after brain injury.

\section{Data handling}

The following data were recorded: general characteristics, including demography, initial GCS score, WFNS grade, time from tracheal intubation to study inclusion, vasopressor therapy, fluid challenges and surgical procedures prior to inclusion. Natraemia, chloraemia, kalaemia, magnesemia, phosphatemia, ionized calcaemia, azotaemia, albuminaemia, osmolarity, lactataemia, arterial gases and haematocrit were measured immediately before and at 6 , 12, 24, 36 and 48 hours after starting the treatment. The total volume of fluid administered and the evolution of ICP were recorded during the study period (48 hours). Episodes of ICH, modifications on the control CT (bleeding, herniation or brain swelling), osmotherapy and/or barbiturate use, transfusion, vasopressor use, time to achieve more than $50 \%$ of goal calories of enteral nutrition, duration of mechanical ventilation, length of ICU stay and mortality rate were also recorded in the ICU. Safety was assessed by recording adverse events.

\section{Definitions}

Strong ion difference (SID) was defined as $\left(\mathrm{Na}^{+}+\mathrm{K}^{+}+\mathrm{Ca}^{2+}\right.$ $\left.+\mathrm{Mg}^{2+}\right)-\left(\mathrm{Cl}^{-}+\right.$lactate $) \mathrm{mEq} / \mathrm{L}$ [22]. Hyperchloraemic metabolic acidosis was defined as SID below $40 \mathrm{mEq} / \mathrm{L}$ associated with chloraemia above $108 \mathrm{mmol} / \mathrm{L}$ according to local laboratory normal ranges.

\section{Endpoints}

The primary endpoint was the occurrence of hyperchloraemic metabolic acidosis within 48 hours. The secondary outcomes were electrolyte status, ICP, rate of ICH episodes, volume of intravenous fluid, duration of vasopressor therapy, duration of mechanical ventilation, length of ICU stay and death in the ICU.

\section{Statistical analysis}

To the best of our knowledge, the incidence of hyperchloraemic acidosis in brain-injured patients has not been documented to date. We have thus performed a post hoc 
analysis of the chloraemia values collected in a study of TBI patients with $\mathrm{ICH}$ receiving HSS [11]. We found a $65 \%$ incidence of hyperchloraemia within the first four days in the ICU before any HSS infusion. The sample size needed to detect a $45 \%$ decrease in the incidence of hyperchloraemic acidosis, assuming a basal rate of $65 \%$ in a two-sided test performed with a statistical power of $85 \%$ and an $\alpha$ risk of 0.05 , was 20 patients in each group in this pilot study. Taking into account exclusions, and in an attempt to keep the power of the study, 42 patients (21 patients in each group) were included.

The full analysis set (FAS) of patients was the primary population used for statistical analysis of efficacy (perprotocol analysis) and was defined as all randomised patients treated with the study drug who did not receive forbidden therapy (HSS infusion). All randomised patients (the intention-to-treat (ITT) population) were analysed for the primary outcome and safety variables.

We first verified that in all patients the incidence of hyperchloraemic acidosis at 48 hours was significantly decreased in the balanced group compared with the control group using Fisher's exact test. Six patients experienced hyperchloraemic acidosis prior to inclusion (four in the saline group and two in the balanced group). We therefore decided a posteriori to perform two complementary sensitivity analyses. The first excluded patients with preexisting hyperchloraemic acidosis, the second censored the preinclusion biological values (SID, chloraemia) and the third consisted of evaluating the effect of balanced solutions on the primary outcome on the basis of a logrank test.

For secondary outcomes, linear mixed models were used with group effect, time effect and interaction between time effect and group effect. We first investigated the interaction between time effect and group effect. For the values with no significant interaction, the mean difference between groups within the study period was provided. For the value with a significant interaction between time effect and group effect, comparisons were performed independently and $\mathrm{P}$ values were calculated at each time point. Residual analysis was used to assess the appropriateness of the models (including normality and homoscedasticity). Nonparametric data are expressed as medians and interquartile ranges (IQRs). Categorical data are expressed as numbers and percentages. $\chi 2$ test, Fisher's exact test and Wilcoxon rank-sum test were used as appropriate.

A subgroup analysis considering severe TBI patients was performed a posteriori using the same analytical strategy. Regarding ICP evolution, subgroup analysis considering the 15 patients with ICH was performed. All statistical tests were two-sided. Statistical analyses were performed using SAS 9.1 statistical software (SAS Institute, Cary, NC, USA).

\section{Results}

\section{Study population}

Of the 42 patients included, 41 were included in the ITT analysis (one consent withdrawal) and 40 were included in the FAS analysis (exclusion of one patient who received HSS infusion within the first six hours; Figure 1). Demographic data are provided in Table 2 (see Additional file, Table S1, for the demographics of the FAS population). The total volume of fluid infusion was not altered by study group (Table 3 ). The total amount of chloride infusion was lower in the balanced group than in the saline group (median $744 \mathrm{mmol}$ (IQR $=572$ to 952$)$ and median $918 \mathrm{mmol}(\mathrm{IQR}=689$ to 1,148), respectively; $P=0.014$ ) (Table 3). Two patients in the saline group and one patient in the balanced group received one bolus of $500 \mathrm{ml}$ of colloid diluted in a saline solution (Gelofusine; B Braun Medical) out of the study protocol, and data from these patients were kept in the statistical analysis.

\section{Efficacy outcomes}

In the ITT population, 19 patients (90\%) in the saline group and 10 patients $(50 \%)$ in the balanced group had hyperchloraemic acidosis within the first 48 hours $(P=$ 0.004). The Kaplan-Meier estimators at hour 48 were $90 \%$ (range $=83 \%$ to $92 \%$ ) in the saline group and $50 \%$ (range $=31 \%$ to $72 \%$ ) in the balanced group, with a hazard ratio (HR) for hyperchloraemic acidosis in the balanced group of 0.24 ( $95 \% \mathrm{CI}=0.10$ to $0.59 ; P=0.003$ ) (Figure 2A).

In the FAS analysis, 18 patients $(90 \%)$ in the saline group and 10 patients (50\%) in the balanced group had hyperchloraemic acidosis within the first 48 hours $(P=$ $0.01)$. The Kaplan-Meier estimators at hour 48 were $90 \%$ (range $=73 \%$ to $98 \%$ ) in the saline group and $50 \%$ (range $=31 \%$ to $72 \%$ ) in the balanced group, with a HR for hyperchloraemic acidosis in the balanced group of 0.28 (95\% CI $=0.11$ to $0.70 ; P=0.006$ ) (Figure 2B). Two sensitivity analyses did not change the results. The HR for hyperchloraemic acidosis in the balanced group was 0.18 (95\% CI $=0.06$ to $0.55 ; P=0.002$ ) when the patients with acidosis prior to inclusion were excluded, and it was 0.25 (95\% $\mathrm{CI}=0.09$ to $0.69 ; P=$ 0.008 ) with a censorship of the biological values (SID, chloraemia) prior to inclusion. In the subgroup of TBI patients, the HR for hyperchloraemic acidosis in the balanced group was $0.30(95 \% \mathrm{CI}=0.12$ to $0.80 ; P=$ 0.015) (Figure $2 \mathrm{C}$ ).

\section{Secondary efficacy outcomes}

The $\mathrm{pH}$ was lower in the saline group than in the balanced group (mean difference $=-0.03(-0.05$ to -0.01$)$; $P=0.004$ ) (Figure 3A). Patients in the saline group had a 


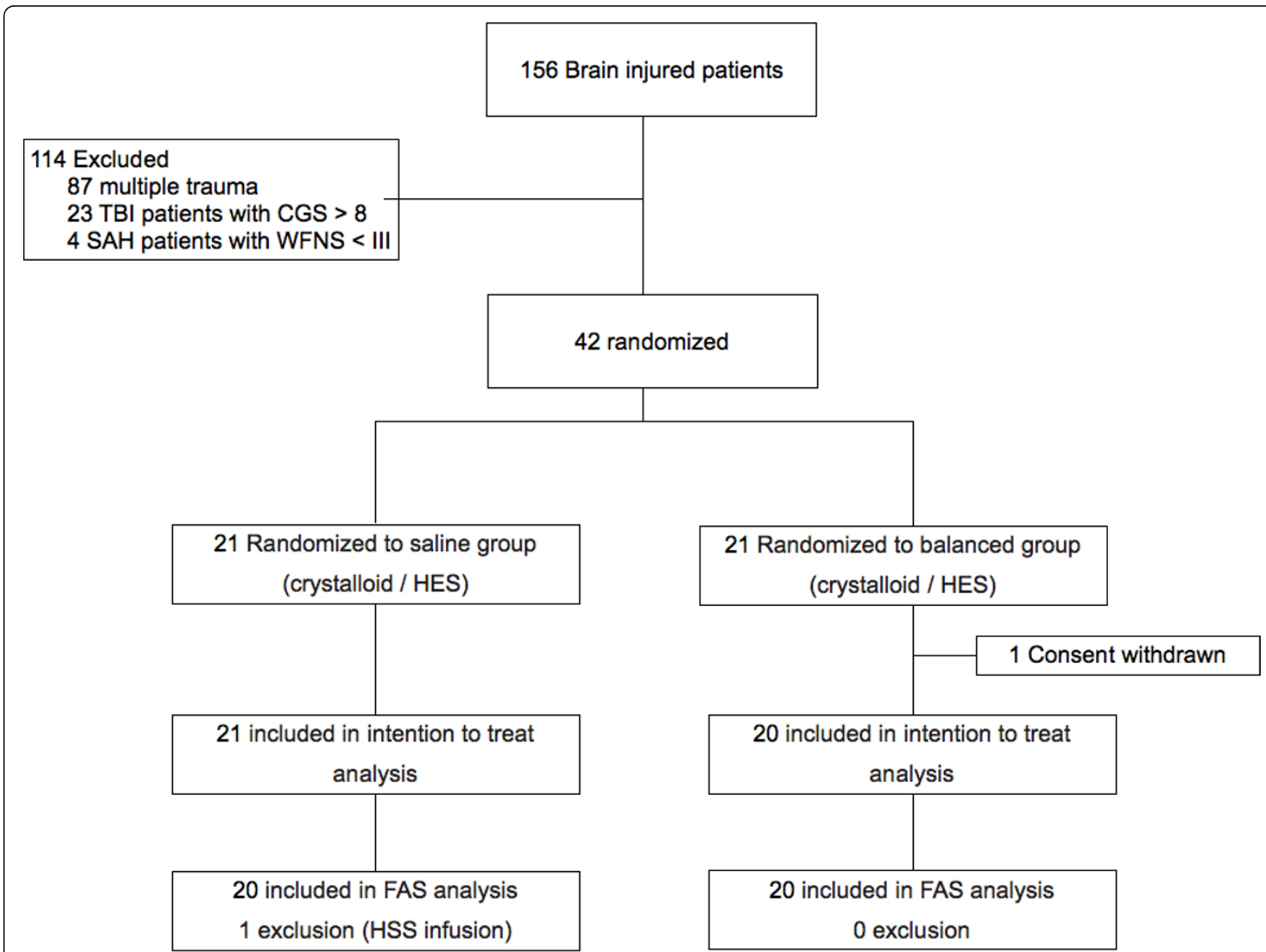

Figure 1 Flowchart of the study. GCS: Glasgow Coma Scale score, FAS: full analysis set; HES: hydroxyethyl starch; HSS: hypertonic saline solution, SAH: subarachnoid haemorrhage, TBI: traumatic brain injury, WFNS: World Federation of Neurological Societies.

lower SID than the balanced group (mean difference $=$ $-1.55 \mathrm{mEq} / \mathrm{L}(-3.09$ to -0.02$) ; P=0.047)$ (Figure $3 \mathrm{~B}$ ). Chloraemia was higher in the saline group than in the balanced group (mean difference $=4.8 \mathrm{mmol} / \mathrm{L}(1.9$ to 7.6); $P=0.002$ ) (Figure 3C). Compared with the balanced group, patients in the saline group had lower phosphataemia (mean difference $=-0.12 \mathrm{mmol} / \mathrm{L}[-0.21$ to -0.04 ); $P=0.008$ ) (Figure 3D). From hour 0 to hour 48, albuminaemia and partial pressure of carbon dioxide in arterial blood $\left(\mathrm{PaCO}_{2}\right)$ were not altered in the saline group compared with the balanced group (Figures $3 \mathrm{E}$ and $3 \mathrm{~F}$ ). The reported differences in the acid-base status between groups were not significantly modified in the subgroup of TBI patients (Additional file, Figure S1). The results for base excess, effective SID, simplified anion gap and corrected anion gap are provided in the Additional file, Table S2. The blood osmolarity was higher in the saline group than in the balanced group (mean difference $=$
$7 \mathrm{mOsmol} / \mathrm{L}$ (1 to 14$) ; P=0.024$ ) (Figure $4 \mathrm{~A}$ ). The natraemia levels were higher in the saline group than in the balanced group (mean difference $=2 \mathrm{mmol} / \mathrm{L}(0$ to 4$) ; P=0.036$ ) (Figure $4 \mathrm{~B})$. ICP was not altered in the study group (mean difference $=4 \mathrm{mmHg}(-1$ to 8 ); $P=0.088$ ) (Figure $4 \mathrm{C}$ ). In the subgroup of TBI patients, blood osmolarity, natraemia and ICP were not altered in the study group (see Additional file, Figure S2). Among the entire population, seven patients (35\%) in the saline group and eight patients (40\%) in the balanced group developed ICH $(P=0.744)$ (Table 4$)$. In the subgroup of patients who developed ICH, ICP was not altered by study group (mean difference $=-4 \mathrm{mmHg}(-11$ to 2 ); $P=0.20$ ). (Additional file, Figure S3).

\section{Safety assessment}

Safety outcomes were assessed for the ITT patient population (Table 4). Decompressive craniectomy was 
Table 2 Baseline characteristics ${ }^{a}$

\begin{tabular}{|c|c|c|c|c|}
\hline \multirow[t]{2}{*}{ Characteristics } & \multicolumn{2}{|c|}{ Intention-to-treat population } & \multicolumn{2}{|l|}{ TBI patients } \\
\hline & Saline $(N=21)$ & Balanced $(N=20)$ & Saline $(N=18)$ & Balanced $(N=18)$ \\
\hline Age (years), median (IQR) & $51(25-68)$ & $49(26-75)$ & $47(28-57)$ & $49(27-77)$ \\
\hline Male, $N(\%)$ & $15(71)$ & $17(85)$ & $13(72)$ & $16(89)$ \\
\hline \multicolumn{5}{|l|}{ Diagnosis, $N(\%)$} \\
\hline Traumatic brain injury & $19(90)$ & $18(90)$ & / & / \\
\hline Subarachnoid haemorrhage & $2(10)$ & $2(10)$ & & \\
\hline Glasgow Coma Scale score on scene, median (IQR) & $8(7-9)$ & $7(6-9)$ & $7(6-8)$ & $7(6-8)$ \\
\hline \multicolumn{5}{|l|}{ Surgical procedures, $N(\%)$} \\
\hline Haematoma/contusion evacuation & $9(43)$ & $4(20)$ & $8(44)$ & $4(22)$ \\
\hline Extraventricular drainage & $2(10)$ & $2(10)$ & 0 & 0 \\
\hline \multicolumn{5}{|l|}{ Transfusion, median (IQR) } \\
\hline Red blood cells, units & $4(19)$ & $3(15)$ & $4(22)$ & $3(17)$ \\
\hline Fresh frozen plasma, units & $3(14)$ & 0 & $3(17)$ & 0 \\
\hline Norepinephrine infusion on admission, $N(\%)$ & $8(38)$ & $7(35)$ & $7(39)$ & $7(39)$ \\
\hline \multicolumn{5}{|l|}{ Fluid infusion prior to inclusion, median (IQR) } \\
\hline Crystalloids ( $\mathrm{NaCl} 0.9 \%), \mathrm{ml}$ & $1000(500-1000)$ & $1000(500-1500)$ & $500(500-1000)$ & $1000(500-1500)$ \\
\hline Colloids, ml & $0(0-500)$ & $0(0-500)$ & $0(0-500)$ & $0(0-500)$ \\
\hline Time from brain injury to inclusion (hours) median (IQR) & $5(3-7)$ & $5(4-12)$ & $4(3-6)$ & $5(4-6)$ \\
\hline \multicolumn{5}{|l|}{ Biological status upon inclusion } \\
\hline Hyperchloraemic acidosis, N (\%) & $4(19)$ & $2(10)$ & $4(22)$ & $2(11)$ \\
\hline Osmolarity (mOsm/L), median (IQR) & $303(295-319)$ & $302(296-319)$ & $306(299-328)$ & $306(300-319)$ \\
\hline Natraemia (mmol/L), median (IQR) & $140(138-142)$ & $139(137-141)$ & $140(138-141)$ & $139(137-141)$ \\
\hline Chloraemia (mmol/L), median (IQR) & $106(104-110)$ & $106(101-107)$ & $106(104-111)$ & $106(100-107)$ \\
\hline Kalemia (mmol/L), median (IQR) & $3.6(3.4-3.9)$ & $3.7(3.5-4.1)$ & $3.7(3.4-3.9)$ & $3.7(3.5-4.1)$ \\
\hline Ionized calcaemia (mmol/L), median (IQR) & $1.10(1.07-1.14)$ & $1.11(1.07-1.18)$ & $1.10(1.08-1.12)$ & $1.11(1.06-1.18)$ \\
\hline Magnesemia (mmol/L), median (IQR) & $0.86(0.72-0.95)$ & $0.81(0.75-0.88)$ & $0.82(0.76-0.92)$ & $0.83(0.73-0.92)$ \\
\hline Phosphoremia (mmol/L), median (IQR) & $0.86(0.67-1.07)$ & $0.85(0.74-1.14)$ & $0.88(0.70-1.10)$ & $0.82(0.73-1.13)$ \\
\hline Lactataemia (mmol/L), median (IQR) & $1.4(1.1-2.1)$ & $1.7(1.1-2.7)$ & $1.5(1.2-2.2)$ & $2.0(1.1-2.7)$ \\
\hline Azotaemia ( $\mu \mathrm{mol} / \mathrm{L})$, median (IQR) & $66(56-73)$ & $67(58-71)$ & $65(56-72)$ & $67(60-71)$ \\
\hline Albuminaemia (g/L), median (IQR) & $36(32-41)$ & $37(34-39)$ & $34(32-41)$ & $36(34-39)$ \\
\hline $\mathrm{pH}$, median (IQR) & $7.38(7.33-7.44)$ & $7.39(7.32-7.45)$ & $7.36(7.31-7.41)$ & $7.39(7.31-7.45)$ \\
\hline SID (mmol/L), median (IQR) & $39(35-42)$ & $39(39-41)$ & $40(37-41)$ & $39(37-41)$ \\
\hline
\end{tabular}

aQR: interquartile range; TBI: traumatic brain injury; Strong lon Difference $(\mathrm{SID})=(\mathrm{Na}+\mathrm{K}+\mathrm{Ca}+\mathrm{Mg})-(\mathrm{Cl}+$ lactate).

Table 3 Fluid administration within the first 48 hours

\begin{tabular}{|c|c|c|c|c|}
\hline & \multicolumn{4}{|c|}{ Median (IQR) } \\
\hline & & $\begin{array}{l}\text { Saline group } \\
(N=20)\end{array}$ & $\begin{array}{l}\text { Balanced group } \\
(N=20)\end{array}$ & $P$-value \\
\hline $\mathrm{Na}$ administration (mmol) & $\mathrm{HO}$ to $\mathrm{H} 48$ & $918(689$ to 1,148$)$ & $840(630$ to 1,050$)$ & 0.228 \\
\hline $\mathrm{Cl}$ administration (mmol) & $\mathrm{HO}$ to $\mathrm{H} 48$ & $918(689$ to 1,148$)$ & 744 (572 to 952) & 0.014 \\
\hline \multirow[t]{4}{*}{ Crystalloids (ml) } & $\mathrm{HO}$ to H6 & $2,000(1,000$ to 2,000$)$ & $1,000(500$ to 2,000$)$ & 0.255 \\
\hline & $\mathrm{H} 6$ to $\mathrm{H} 24$ & $2,000(1,500$ to 2,000$)$ & $1,500(1,500$ to 2,000$)$ & 0.530 \\
\hline & $\mathrm{H} 24$ to $\mathrm{H} 48$ & $2,000(1,500$ to 2,000$)$ & $2,000(1,500$ to 2,000$)$ & 0.755 \\
\hline & $\mathrm{HO}$ to $\mathrm{H} 48$ & $5,000(4,500$ to 6,000$)$ & $5,000(4,000$ to 6,000$)$ & 0.448 \\
\hline \multirow[t]{4}{*}{ Hydroxyethyl starch solutions (ml) } & $\mathrm{HO}$ to $\mathrm{H} 6$ & $0(0$ to 500$)$ & $0(0$ to 500$)$ & 0.613 \\
\hline & $\mathrm{H} 6$ to $\mathrm{H} 24$ & $0(0$ to 500$)$ & $0(0$ to 500$)$ & 0.563 \\
\hline & $\mathrm{H} 24$ to $\mathrm{H} 48$ & $0(0$ to 0$)$ & $500(0$ to 1,000$)$ & 0.060 \\
\hline & $\mathrm{HO}$ to $\mathrm{H} 48$ & $500(0$ to 1,500$)$ & $1,000(500$ to 1,500$)$ & 0.228 \\
\hline
\end{tabular}




\section{A. Intention to treat analysis}

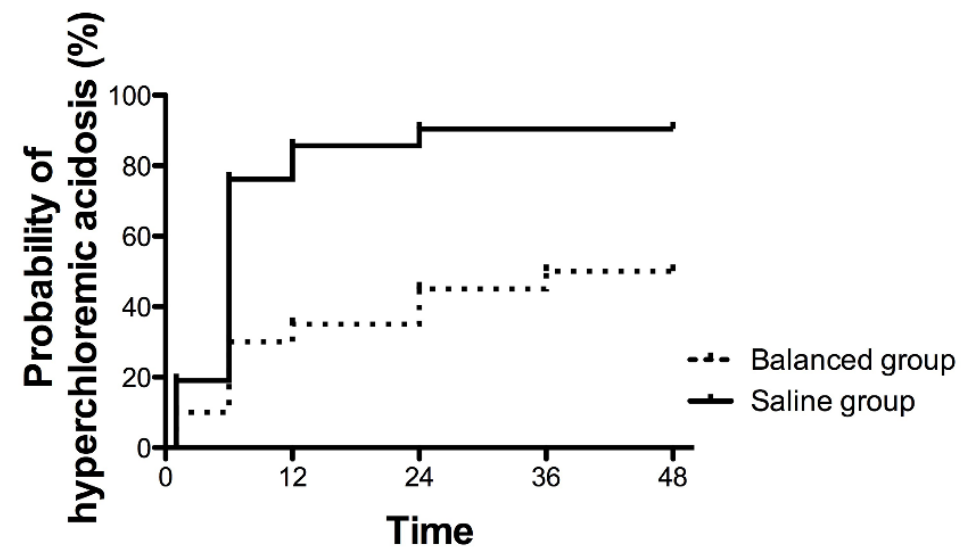

B. Full assessment set analysis

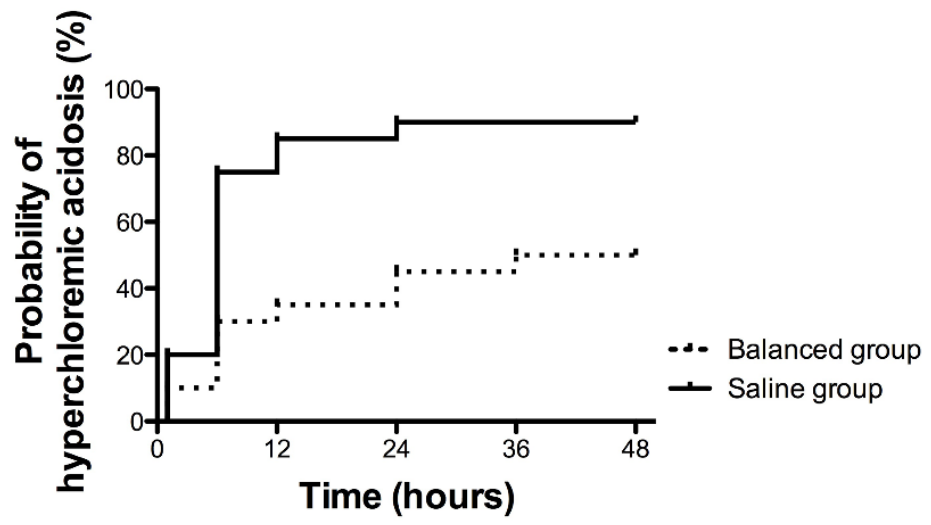

\section{Sub-group of patients with traumatic brain-injured patients}

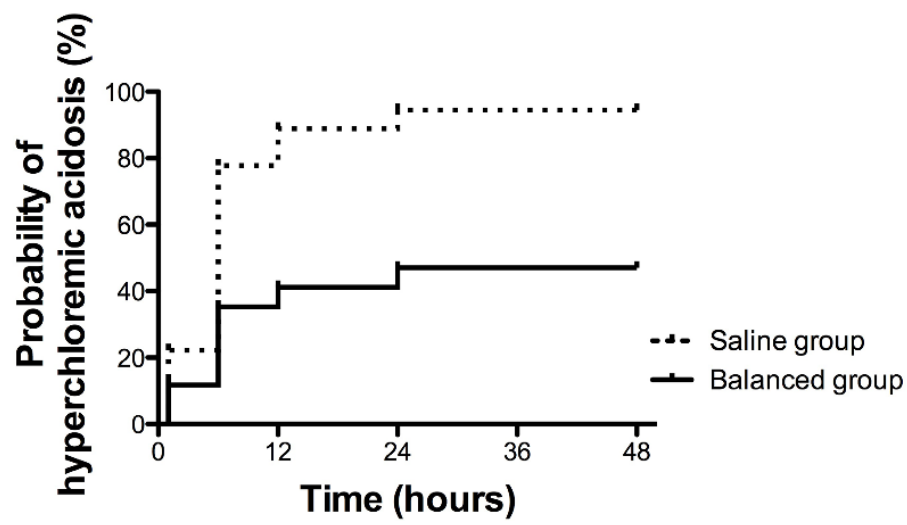

Figure 2 Kaplan-Meier curves for hyperchloraemic acidosis. Hyperchloraemic acidosis was defined as the association of hyperchloraemia (>108 mmol/L) with strong ion difference (SID) $(<40 \mathrm{mmol} / \mathrm{L}) . \mathrm{SID}=(\mathrm{Na}+\mathrm{K}+\mathrm{Ca}+\mathrm{Mg})-(\mathrm{Cl}+$ lactate). Na; sodium, $\mathrm{K} ;$ potassium; Ca: calcium; Mg: magnesium; Cl: chloride. 


\section{B.}
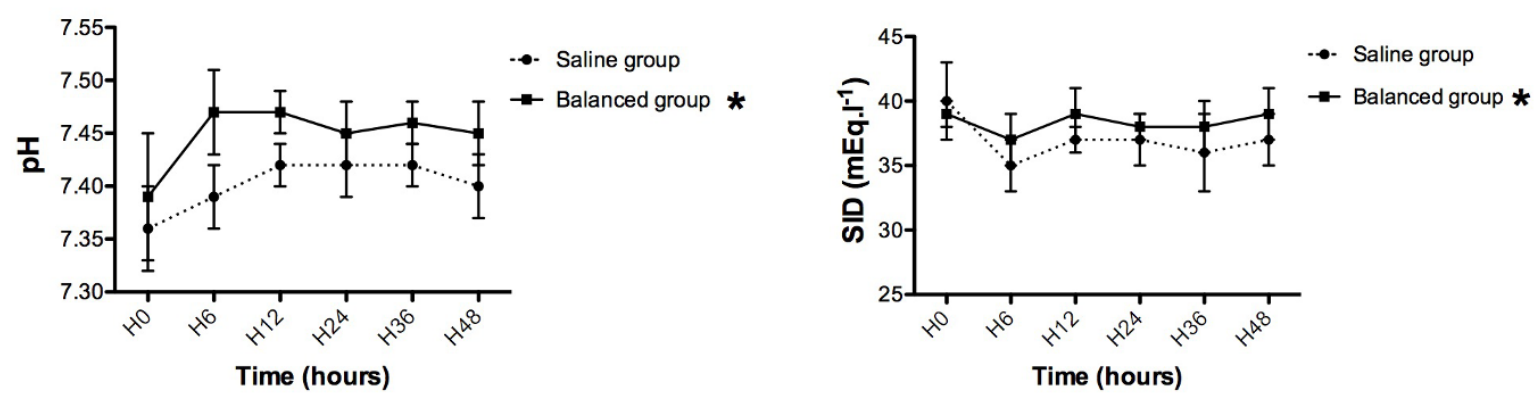

C.
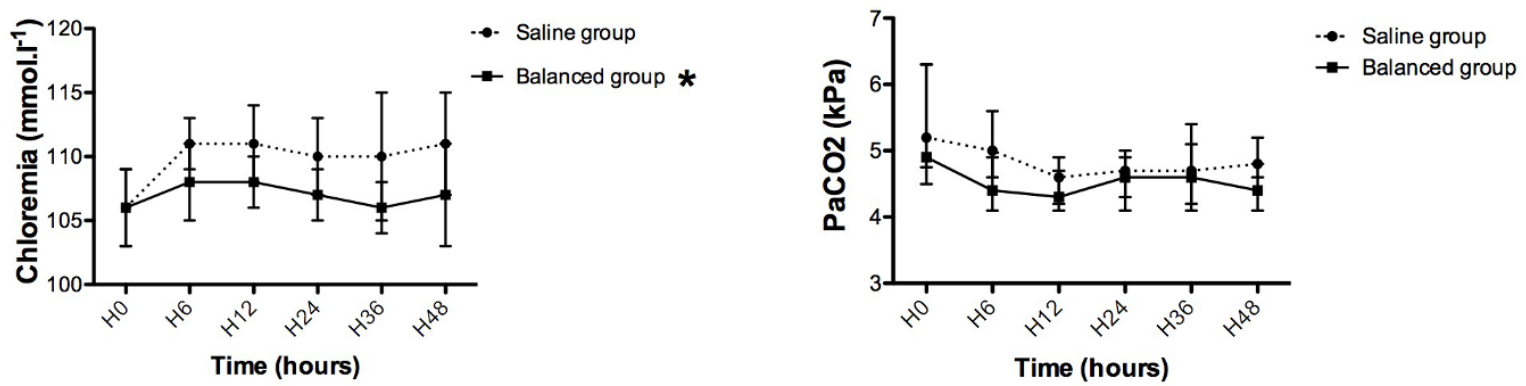

E.
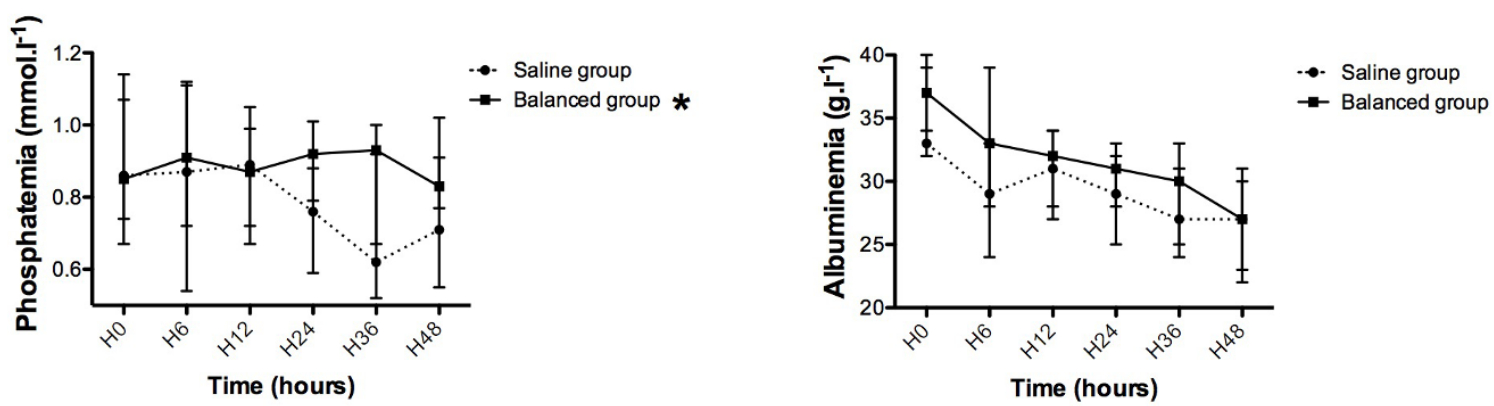

Figure 3 Time course of acid-base status in the saline group and the balanced group. Hyperchloraemic acidosis was defined as the association of hyperchloraemia (>108 mmol/L) with strong ion difference (SID) $<40 \mathrm{mmol} / \mathrm{L}$. SID $=(\mathrm{Na}+\mathrm{K}+\mathrm{Ca}+\mathrm{Mg})-(\mathrm{Cl}+\mathrm{lactate})$. $\{\mathrm{AU}$ : OK to delete Kaplan? OK Or are words missing?\}According to Stewart et al. (A) pH is independently influenced by three biological values: first, the SID (B) and chloraemia (C); second, the total weak acid concentration composed of phosphor (D) and albumin (E); and third, the partial pressure of carbon dioxide in arterial blood $\left(\mathrm{PaCO}_{2}\right)$ (F). Results are given as medians (IQR). ${ }^{*} \mathrm{P}<0.05$ versus saline group (significant group effect). Na: sodium, K: potassium, Ca: calcium, Mg: magnesium, Cl: chloride.

performed before ICU admission in one patient (5\%) in the balanced group compared with one patient (5\%) in the saline group $(P=0.972)$ (Table 4$)$. Three patients (14\%) in the saline group died (two with $\mathrm{ICH}(10 \%)$ and one due to care withdrawal $(5 \%))$, compared with five patients $(25 \%)$ in the balanced group (two patients with ICH $(10 \%)$ and three patients due to care withdrawal $(15 \%))(P=0.387)$. No patients died during the study
periodThere is no need to provide this information since it is stated that 3 patients died in the saline group and five in the other group.

\section{Discussion}

The present study shows that balanced solutions, in comparison with isotonic saline solutions, reduced the occurrence of hyperchloraemic acidosis in brain-injured 


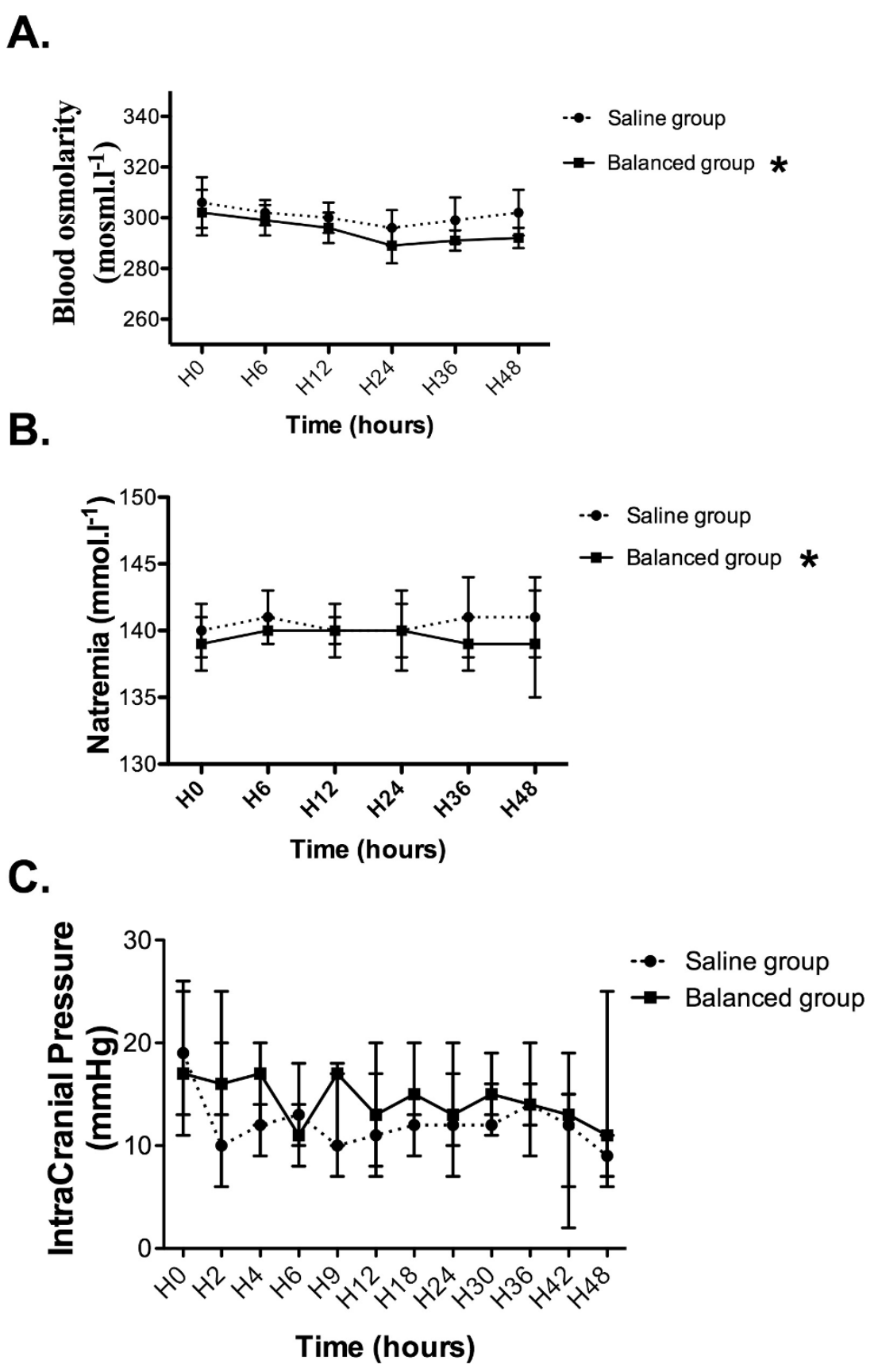

Figure 4 Time course of (A) blood osmolarity, (B) natraemia and (C) intracranial pressure in the saline group and the balanced group. Results are given as medians (IQR). ${ }^{*} P<0.05$ versus saline group (significant group effect).

patients. Balanced solutions were not associated with ICP alteration or ICH episodes.

According to Stewart et al., hydrogen ion concentration $(\mathrm{pH})$ is independently influenced by three biological variables: (1) $\mathrm{PaCO}_{2}$, (2) total weak acid concentration (labelled $\mathrm{A}_{\text {tot }}$ ) composed of phosphate and albumin and (3) SID corresponding to the difference between strong cations and strong anions [22]. According to Stewart et al.'s concept, sodium chloride solutions are responsible for metabolic acidosis through a decrease in SID [23-25]. Thus, administration of drugs with sodium chloride excipient (such as cloxacillin, midazolam or fentanyl) participate in the decrease in SID, which could partially explain the $50 \%$ rate of hyperchloraemic acidosis in the balanced group. The concentration of chloride in $0.9 \%$ saline solutions surpasses the normal ranges for blood 
Table 4 Safety assessment ${ }^{\mathrm{a}}$

\begin{tabular}{|c|c|c|c|c|c|c|}
\hline \multirow[t]{2}{*}{ Outcomes } & \multicolumn{3}{|l|}{ ITT population } & \multicolumn{3}{|l|}{ TBI patients } \\
\hline & $\begin{array}{l}\text { Saline } \\
(N=21)\end{array}$ & $\begin{array}{l}\text { Balanced } \\
(N=20)\end{array}$ & $\begin{array}{l}P \text { - } \\
\text { value }\end{array}$ & $\begin{array}{l}\text { Saline } \\
(N=18)\end{array}$ & $\begin{array}{l}\text { Balanced } \\
(N=18)\end{array}$ & $\begin{array}{l}P \text { - } \\
\text { value }\end{array}$ \\
\hline Patients with an episode of $\mathrm{ICH}, n(\%)$ & $8(38)$ & $8(40)$ & 0.905 & $6(33)$ & $8(44)$ & 0.494 \\
\hline Number of $\mathrm{ICH}$ episode per patient, mean \pm SD & $3 \pm 5$ & $1 \pm 2$ & 0.912 & $3 \pm 6$ & $1 \pm 1$ & 0.957 \\
\hline \multicolumn{7}{|l|}{ Management of $\mathrm{ICH}, n(\%)$} \\
\hline Osmotherapy (mannitol) & $8(38)$ & $7(35)$ & 0.837 & $6(33)$ & $7(39)$ & 0.729 \\
\hline Barbiturate & $7(33)$ & $6(30)$ & 0.819 & $5(28)$ & $6(33)$ & 0.718 \\
\hline Decompressive craniectomy & $1(5)$ & $1(5)$ & 0.972 & $1(6)$ & $1(6)$ & 1 \\
\hline Therapeutic hypothermia & $0(0)$ & $0(0)$ & 1 & $0(0)$ & $0(0)$ & 1 \\
\hline \multicolumn{7}{|l|}{ Modifications on CT, $n$ (\%) } \\
\hline Bleeding & $1(5)$ & $2(10)$ & 0.52 & $1(6)$ & $2(11)$ & 1 \\
\hline Herniation & $0(0)$ & $1(5)$ & 0.3 & $0(0)$ & $1(6)$ & 1 \\
\hline Brain swelling & $4(19)$ & $2(10)$ & 0.413 & $3(17)$ & $1(6)$ & 0.602 \\
\hline \multicolumn{7}{|l|}{ In ICU transfusion, $n(\%)$} \\
\hline Red blood cells & $7(33)$ & $4(20)$ & 0.336 & $7(39)$ & $4(22)$ & 0.278 \\
\hline Fresh frozen plasma & $5(24)$ & $1(5)$ & 0.089 & $5(28)$ & $1(6)$ & 0.178 \\
\hline Duration of norepinephrine infusion, days, median (IQR) & $5(2$ to 7$)$ & $4(2$ to 7$)$ & 0.676 & 3 (1 to 4$)$ & $4(2$ to 6$)$ & 0.503 \\
\hline Potassium administration, $n$ (\%) & $13(60)$ & $11(55)$ & 0.654 & $10(56)$ & $10(56)$ & 1 \\
\hline Calcium administration, $n$ (\%) & $4(20)$ & $2(10)$ & 0.661 & $3(17)$ & $2(11)$ & 1 \\
\hline \multicolumn{7}{|l|}{ Azotaemia, (mol/L), median (IQR) } \\
\hline Day 1 & 59 (56 to 68$)$ & $60(50$ to 67$)$ & 0.879 & 64 (57 to 74) & 67 (59 to 71$)$ & 0.975 \\
\hline Day 2 & 60 (54 to 65$)$ & 56 (46 to 64) & 0.289 & 58 (56 to 67) & $62(52$ to 68$)$ & 0.734 \\
\hline \multicolumn{7}{|l|}{ Diuresis, L/day, median (IQR) } \\
\hline Day 1 & $\begin{array}{l}1.7(1.2 \text { to } \\
2.5)\end{array}$ & $\begin{array}{l}1.6(1.1 \text { to } \\
2.1)\end{array}$ & 0.551 & $\begin{array}{l}1.6(1.2 \text { to } \\
2.3)\end{array}$ & $\begin{array}{l}1.6(1.2 \text { to } \\
2.0\end{array}$ & 0.756 \\
\hline Day 2 & $\begin{array}{l}1.6(1.0 \text { to } \\
2.6)\end{array}$ & $\begin{array}{l}1.5(1.0 \text { to } \\
2.0)\end{array}$ & 0.845 & $\begin{array}{l}1.6(1.0 \text { to } \\
2.2)\end{array}$ & $\begin{array}{l}1.5(1.0 \text { to } \\
2.0)\end{array}$ & 0.851 \\
\hline $\begin{array}{l}\text { Time to achieve }>50 \% \text { of goal calories of enteral nutrition, days, } \\
\text { median (IQR) }\end{array}$ & 4 (3 to 5$)$ & $3(3$ to 11$)$ & 0.911 & 4 (3 to 6) & $3(3$ to 11$)$ & 1 \\
\hline Duration of mechanical ventilation, days, median (IQR) & $12(5$ to 18$)$ & $12(8$ to 19$)$ & 0.823 & $10(2$ to 18$)$ & $10(7$ to 19$)$ & 0.76 \\
\hline ICU length of stay, days, median (IQR) & 19 (10 to 24$)$ & 16 (8 to 21$)$ & 0.521 & 18 (10 to 24$)$ & 14 (7 to 21$)$ & 0.591 \\
\hline Death in ICU, $n(\%)$ & $3(14)$ & $5(25)$ & 0.387 & $2(11)$ & $5(28)$ & 0.402 \\
\hline Refractory $\mathrm{ICH}$ & $2(10)$ & $2(10)$ & & $1(6)$ & $2(11)$ & \\
\hline Care withdrawal & $1(5)$ & $3(15)$ & & $1(6)$ & $3(17)$ & \\
\hline
\end{tabular}

${ }^{a} \mathrm{CT}$ : computed tomography, ICH: intracranial hypertension, IQR: interquartile range, ITT: intention to treat; TBI: traumatic brain injury.

chloraemia [26]. A correlation between hyperchloraemia and base excess has been described in patients undergoing major surgery [16]. Balanced solutions reduce the risk of hyperchloraemic acidosis in elderly patients undergoing major surgery $[27,28]$. Our results reveal that balanced solutions decrease the risk of hyperchloraemic acidosis in patients with severe brain injury.

At this time, there is increasing evidence that chloriderich solutions alter the outcomes of critical ill patients [9]. In animal models, chloride-rich solutions decreased the glomerular filtration rate by inducing renal vasoconstriction $[29,30]$. In a before-after study, the restriction of chloride-rich solutions was associated with a decrease in kidney failure in critically ill patients [10]. Saline-rich solutions alter the coagulation cascade and increase intraoperative blood loss when compared with balanced solutions [31-34]. Hyperchloraemic acidosis also decreases gastric and pyloric motility and could reduce gastric mucosal perfusion [28]. In the present study, balanced solutions prevented hyperchloraemic acidosis but altered neither kidney function (assessed by diuresis and azotaemia) nor gut motility (assessed by the time to achieve more than $50 \%$ of enteral nutrition goal calories).

Prevention of hypo-osmolarity is a major goal for the prevention and treatment of $\mathrm{ICH}$. Indeed, hypo-osmolarity induces brain ischaemia resulting from the swelling of perivascular astrocytic cells and also increases ICP and the volume of brain injury [35]. Thus, administration of hypoosmolar solutions should be avoided in brain-injured patients $[6,7]$. The isotonicity of the balanced solutions may authorize their utilization in the neuro-ICU, but few 
data are available in this setting to date. In the present study, the balanced solutions failed to induce hyperosmolarity, and the blood osmolarity was lower in the balanced group than in the saline group. This lower osmolarity in the balanced group may prove important because the maintenance of normal osmolarity is an asset when caring for the brain-injured patients. However, neither ICP evolution nor the rate of $\mathrm{ICH}$ were different between the study groups. These results could be explained by the impact of balanced solutions on chloraemia, which is a key regulator of cell volume [10]. Chloride ion efflux prevents cell swelling in hypotonic media [12,36]. The lower chloraemia observed in the balanced group could have increased the phenomenon of chloride ion efflux, limiting brain swelling despite decreased osmolarity compared with the saline group. According to this hypothesis, it has previously been described that a sodium lactate-based hyperosmolar solution more significantly decreased ICP than an equivalent osmotic load of chloride-rich solution [35]. Prevention of hyperchloraemia appears to be an asset for the prevention of ICH in patients with severe brain injury.

There is a controversy about the safety of HES, particularly regarding its effects on coagulation [37]. These concerns could prove to be important in the setting of brain-injured patients. Recently, the Neuro-Intensive Care and Emergency Medicine (NICEM) Section of the European Society of Intensive Care Medicine consensus document stated that HES is not recommended in the context of brain injury [38]. However, this consensus statement was not available when our study started. Moreover, the doses of HES used in each group in our study were below the maximum daily threshold of $20 \mathrm{ml} / \mathrm{kg} /$ day.

This study has several limitations. First, given the small number of included SAH patients, the conclusions are valid mainly for the TBI patients. Second, we did not report any differences between groups regarding side effects of hyperchloraemic acidosis. Third, the reported biological differences may not be clinically relevant. Prolonged infusion of $0.9 \%$ saline solution may alter clinical outcomes. Fourth, the balanced solution did not alter neurological recovery, and we cannot rule out the theoretical issue regarding the risk of $\mathrm{ICH}$ with balanced solutions. However, this pilot study was not powered to evaluate these endpoints.

\section{Conclusions}

The use of balanced solutions reduces the incidence of hyperchloraemic acidosis in brain-injured patients. ICP evolution and the rate of $\mathrm{ICH}$ in brain-injured patients did not appear to be different between groups. The safety and impact of balanced solutions on neurological recovery, as well as the potential side effects of balanced solutions, should be investigated in a large, randomised trial comparing balanced solutions and isotonic saline solutions in TBI patients.

\section{Key messages}

- Balanced solutions decrease the incidence of hyperchloraemic acidosis in patients with severe brain injury compare with saline solutions.

- Balanced solutions decrease natraemia and blood osmolarity in patients with severe brain injury.

- Larger studies are required to investigate the effects of balanced solutions on brain swelling and neurological recovery.

\section{Additional material}

Additional file 1: Enteral Nutrition Protocol. Table S1. Baseline characteristics. Table S2. Time evolution of biological values within the first 48 hours Simplified anion gap $(s A G)=\mathrm{Na}-\left(\mathrm{Cl}+\mathrm{HCO}_{3}\right)$. Corrected anion gap $(\mathrm{cAG})=\mathrm{sAG}+0.25 \times(40-$ albumin $)$. Effective strong ion difference effective $(\mathrm{SIDe})=\mathrm{HCO}_{3}+$ albumin $\times(0.123 \times \mathrm{pH}-0.631)$ + phosphor $\times(0.309 \times \mathrm{pH}-0.469)$. Data are expressed as median (IQR) \#\#Data with a significant interaction between time effect and group effect, comparisons were performed independently for each time point , and $\mathrm{P}$ values were provided at each time point. Figure S1. Time course of acid-base status in TBI patients. Results are given as median (IQR). ${ }^{*} \mathrm{P}<0.05$ versus saline group (significant group effect). TBI: traumatic brain injury. Figure S2. Time course of (A) blood osmolarity, (B) natraemia and (C) intracranial pressure in traumatic braininjured patients. Results are given as medians (IQR). Figure S3. Time course of intracranial pressure in brain-injured patients who developed intracranial hypertension. Results are given as medians (IQR).

\section{Abbreviations}

CT: computed tomography; GCS: Glasgow Coma Scale; HES: hydroxyethyl starch; HSS: hypertonic saline solution; ICH: intracranial hypertension; ICP: intracranial pressure; SAH: subarachnoid haemorrhage; SID: strong ion difference; TBI: traumatic brain injury; WFNS: World Federation of Neurological Societies.

\section{Competing interests}

Karim Asehnoune and Yvonnick Blanloeil have received honoraria from B Braun Medical for public speaking. The other authors have no conflicts of interest to disclose.

\section{Authors' contributions}

All of the authors participated in the study management, data collection and interpretation of data. $O L, A R, C L, Y B$ and $K A$ were responsible for the conception and design of the study, interpretation of data and/or writing of the report. RC, ER, PJM, RD, AMC and CP were responsible for data collection, data interpretation and/or writing the report. CV performed statistical analysis. LF managed the blinding and the safety of the study solutions. All authors had full access to all of the data in the study and participated in the revision of the manuscript. All authors read and approved the manuscript for publication.

\section{Acknowledgements}

We gratefully acknowledge Delphine Flattres for her critical help with the study and the nurses and medical team of the Surgical Intensive Care Units of Nantes University Hospital for technical assistance. This work was supported by B-BRAUN MEDICAL. B-BRAUN MEDICAL provided the solutions but was not involved in the study design, patient recruitment, data collection and analysis, report writing and publication. The University Hospital of Nantes (UHN) sponsored the study. UHN stored the data, ensured the monitoring of the study. The biostatistics unit (Christelle 
Volteau) of UHN performed the statistical analysis. The local pharmacist (Laurent Flet) blinded the solutions. The results were presented (abstract) at the 53th congress of the French Congress of Anaesthesia and Critical Care Medicine (SFAR 2011).

\section{Authors' details}

${ }^{1}$ Pôle Anesthésie-Réanimations, Service d'anesthésie réanimation Hôtel-Dieu, CHU Nantes, F-44000 Nantes, France. ²Pôle Anesthésie-Réanimations, Service d'anesthésie réanimation Hôpital Laennec, CHU Nantes, F-44000 Nantes, France. ${ }^{3}$ Pôle Anesthésie-Réanimations, Service de réanimation médicale polyvalente, CHU Nantes, F-44000 Nantes, France. ${ }^{4}$ Pôle Pharmacie, Service HOTEL-DIEU-Essais cliniques, CHU Nantes, F-44000 Nantes, France. ${ }^{5}$ Cellule de Biostatistiques-Cellule de promotion à la recherche clinique, CHU Nantes, F-44000 Nantes, France.

Received: 8 December 2012 Revised: 14 December 2012

Accepted: 19 April 2013 Published: 19 April 2013

\section{References}

1. Mathers CD, Loncar D: Projections of global mortality and burden of disease from 2002 to 2030. PLoS Med 2006, 3:e442.

2. Chesnut RM, Marshall LF, Klauber MR, Blunt BA, Baldwin N, Eisenberg HM, Jane JA, Marmarou A, Foulkes MA: The role of secondary brain injury in determining outcome from severe head injury. J Trauma 1993, 34:216-222.

3. Dutton RP, Stansbury LG, Leone S, Kramer E, Hess JR, Scalea TM: Trauma mortality in mature trauma systems: Are we doing better? An analysis of trauma mortality patterns, 1997-2008. J Trauma 2010, 69:620-626.

4. Simma B, Burger R, Falk M, Sacher P, Fanconi S: A prospective, randomized, and controlled study of fluid management in children with severe head injury: lactated Ringer's solution versus hypertonic saline. Crit Care Med 1998, 26:1265-1270.

5. Shackford SR, Bourguignon PR, Wald SL, Rogers FB, Osler TM, Clark DE: Hypertonic saline resuscitation of patients with head injury: a prospective, randomized clinical trial. J Trauma 1998, 44:50-58.

6. Bederson JB, Connolly ES Jr, Batjer HH, Dacey RG, Dion JE, Diringer MN, Duldner JE Jr, Harbaugh RE, Patel AB, Rosenwasser RH; American Heart Association: Guidelines for the management of aneurysmal subarachnoid hemorrhage: a statement for healthcare professionals from a special writing group of the Stroke Council, American Heart Association. Stroke 2009, 40:994-1025.

7. Brain Trauma Foundation American Association of Neurological Surgeons Congress of Neurological Surgeons: Guidelines for the management of severe traumatic brain injury: 3rd edition. J Neurotrauma 2007, 24(Suppl 1):S1-S106.

8. Yunos NM, Bellomo R, Story D, Kellum J: Bench-to-bedside review: chloride in critical illness. Crit Care 2010, 14:226.

9. Yunos NM, Kim IB, Bellomo R, Bailey M, Ho L, Story D, Gutteridge GA, Hart GK: The biochemical effects of restricting chloride-rich fluids in intensive care. Crit Care Med 2011, 39:2419-2424.

10. Yunos NM, Bellomo R, Hegarty C, Story D, Ho L, Bailey M: Association between a chloride-liberal vs chloride-restrictive intravenous fluid administration strategy and kidney injury in critically ill adults. JAMA 2012, 308:1566-1572.

11. Roquilly A, Mahe PJ, Demeure Dit Latte D, Loutrel O, Champin P, Di Falco C, Courbe A, Buffenoir K, Hamel O, Lejus C, Sebille V, Asehnoune K: Continuous controlled-infusion of hypertonic saline solution in traumatic braininjured patients: a 9-year retrospective study. Crit Care 2011, 15:R260

12. Jentsch TJ, Stein V, Weinreich F, Zdebik AA: Molecular structure and physiological function of chloride channels. Physiol Rev 2002, 82:503-568.

13. Williams EL, Hildebrand KL, McCormick SA, Bedel MJ: The effect of intravenous lactated Ringer's solution versus $0.9 \%$ sodium chloride solution on serum osmolality in human volunteers. Anesth Analg 1999, 88:999-1003

14. Wilkes NJ, Woolf RL, Powanda MC, Gan TJ, Machin SJ, Webb A, Mutch M, Bennett-Guerrero E, Mythen M: Hydroxyethyl starch in balanced electrolyte solution (Hextend)-pharmacokinetic and pharmacodynamic profiles in healthy volunteers. Anesth Analg 2002, 94:538-544.

15. Scheingraber S, Rehm M, Sehmisch C, Finsterer U: Rapid saline infusion produces hyperchloremic acidosis in patients undergoing gynecologic surgery. Anesthesiology 1999, 90:1265-1270.
16. Waters JH, Miller LR, Clack S, Kim JV: Cause of metabolic acidosis in prolonged surgery. Crit Care Med 1999, 27:2142-2146.

17. McFarlane C, Lee A: A comparison of Plasmalyte 148 and $0.9 \%$ saline for intra-operative fluid replacement. Anaesthesia 1994, 49:779-781.

18. Audibert G, Hoche J, Baumann A, Mertes PM: [Water and electrolytes disorders after brain injury: mechanism and treatment] [in French]. Ann Fr Anesth Reanim 2012, 31:e109-e115.

19. Jayakumar AR, Norenberg MD: The Na-K-Cl co-transporter in astrocyte swelling. Metab Brain Dis 2010, 25:31-38.

20. Lepelletier D, Roquilly A, Demeure Dit Latte D, Mahe PJ, Loutrel O, Champin P, Corvec S, Naux E, Pinaud M, Lejus C, Asehnoune K: Retrospective analysis of the risk factors and pathogens associated with early-onset ventilator-associated pneumonia in surgical-ICU head-trauma patients. J Neurosurg Anesthesiol 2010, 22:32-37.

21. Andrews PJ, Citerio G, Longhi L, Polderman K, Sahuquillo J, Vajkoczy P, Neuro-Intensive Care and Emergency Medicine (NICEM) Section of the European Society of Intensive Care Medicine: NICEM consensus on neurological monitoring in acute neurological disease. Intensive Care Med 2008, 34:1362-1370.

22. Stewart PA: Modern quantitative acid-base chemistry. Can J Physiol Pharmacol 1983, 61:1444-1461.

23. Blanloeil Y, Roze B, Rigal JC, Baron JF: [Hyperchloremic acidosis during plasma volume replacement] [in French]. Ann Fr Anesth Reanim 2002, 21:211-220.

24. Kellum JA: Clinical review: reunification of acid-base physiology. Crit Care 2005, 9:500-507.

25. Kellum JA: Determinants of blood $\mathrm{pH}$ in health and disease. Crit Care 2000, 4:6-14.

26. Wakim KG: "Normal" 0.9 per cent salt solution is neither "normal" nor physiological. JAMA 1970, 214:1710

27. Gan TJ, Bennett-Guerrero E, Phillips-Bute B, Wakeling H, Moskowitz DM, Olufolabi Y, Konstadt SN, Bradford C, Glass PS, Machin SJ, Mythen MG, the Hextend Study Group: Hextend ${ }^{\circledR}$, a physiologically balanced plasma expander for large volume use in major surgery: a randomized phase III clinical trial. Anesth Analg 1999, 88:992-998.

28. Wilkes NJ, Woolf R, Mutch M, Mallett SV, Peachey T, Stephens R, Mythen MG: The effects of balanced versus saline-based hetastarch and crystalloid solutions on acid-base and electrolyte status and gastric mucosal perfusion in elderly surgical patients. Anesth Analg 2001, 93:811-816.

29. Wilcox CS: Regulation of renal blood flow by plasma chloride. J Clin Invest 1983, 71:726-735.

30. Todd SR, Malinoski D, Muller PJ, Schreiber MA: Lactated Ringer's is superior to normal saline in the resuscitation of uncontrolled hemorrhagic shock. J Trauma 2007, 62:636-639.

31. Roche AM, James MFM, Bennett-Guerrero E, Mythen MG: A head-to-head comparison of the in vitro coagulation effects of saline-based and balanced electrolyte crystalloid and colloid intravenous fluids. Anesth Analg 2006, 102:1274-1279.

32. Roche AM, James MFM, Grocott MPW, Mythen MG: Coagulation effects of in vitro serial haemodilution with a balanced electrolyte hetastarch solution compared with a saline-based hetastarch solution and lactated Ringer's solution. Anaesthesia 2002, 57:950-955.

33. Kiraly LN, Differding JA, Enomoto TM, Sawai RS, Muller PJ, Diggs B, Tieu BH, Englehart MS, Underwood S, Wiesberg TT, Schreiber MA: Resuscitation with normal saline (NS) vs. lactated Ringer's (LR) modulates hypercoagulability and leads to increased blood loss in an uncontrolled hemorrhagic shock swine model. J Trauma 2006, 61:57-65.

34. Martin G, Bennett-Guerrero E, Wakeling H, Mythen MG, el-Moalem H, Robertson K, Kucmeroski D, Gan TJ: A prospective, randomized comparison of thromboelastographic coagulation profile in patients receiving lactated Ringer's solution, $6 \%$ hetastarch in a balanced-saline vehicle, or $6 \%$ hetastarch in saline during major surgery. J Cardiothorac Vasc Anesth 2002, 16:441-446.

35. Ichai C, Armando G, Orban JC, Berthier F, Rami L, Samat-Long C, Grimaud D, Leverve X: Sodium lactate versus mannitol in the treatment of intracranial hypertensive episodes in severe traumatic brain-injured patients. Intensive Care Med 2009, 35:471-479.

36. Adrogué HJ, Madias NE: Hyponatremia. N Engl J Med 2000, 342:1581-1589.

37. Gattas DJ, Dan A, Myburgh J, Billot L, Lo S, Finfer S: the CHEST Management Committee: Fluid resuscitation with $6 \%$ hydroxyethyl 
starch (130/0.4) in acutely ill patients: an updated systematic review and meta-analysis. Anesth Analg 2012, 114:159-169.

38. Reinhart K, Perner A, Sprung CL, Jaeschke R, Schortgen F, Groeneveld ABJ, Beale R, Hartog CS, European Society of Intensive Care Medicine:

Consensus statement of the ESICM task force on colloid volume therapy in critically ill patients. Intensive Care Med 2012, 38:368-383.

\section{doi:10.1186/cc12686}

Cite this article as: Roquilly et al:: Balanced versus chloride-rich solutions for fluid resuscitation in brain-injured patients: a randomised double-blind pilot study. Critical Care 2013 17:R77.

Submit your next manuscript to BioMed Central and take full advantage of:

- Convenient online submission

- Thorough peer review

- No space constraints or color figure charges

- Immediate publication on acceptance

- Inclusion in PubMed, CAS, Scopus and Google Scholar

- Research which is freely available for redistribution

Submit your manuscript at www.biomedcentral.com/submit 